\section{De las mujeres al género y más allá: las entretejidas trayectorias de la historia de las mujeres y los estudios feministas*}

\section{(4) Eileen Boris}

University of California Santa Barbara, Profesora titular de Estudios Feministas eboris@ucsb.edu
* Artículo traducido por Martina Altalef / martina.altalef@gmail.com
Fecha de recepción: 01/08/2017. Fecha de aceptación: 01/12/2017.

\title{
Resumen
}

En la década de 1980 los Estudios de las mujeres o con perspectiva de género y la historia de las mujeres o con perspectiva de género parecían estar más cercanos de lo que subsiguientemente han devenido. Aquí, me pregunto, ¿pueden los Estudios de las mujeres o con perspectiva de género o feministas ser historizados y puede la

\section{Palabras clave:}

Estudios de género o de las mujeres; historia; teoría queer; teoría feminista; tiempo; archivo. historia ser teorizada por la teoría queer y feminista de un modo que ilumine el pasado en sus propios términos y no meramente hable del presente o del futuro? Considero las prácticas de citado y subcampos a la luz de tendencias intelectuales más amplias, especialmente el trabajo del feminismo negro. Al explorar esta pregunta, me vuelvo hacia cómo cada campo piensa acerca del tiempo y el archivo, términos que funcionan como analíticos para iluminar interrogantes sobre poder y autoridad, identidad y agencia, pero desplegados por investigadorxs feministas interdisciplinarixs de formas que no son siempre legibles para historiadorxs tradicionales. Concluyo tanto con finales de reconciliación y alternativa a la luz de los ataques sobre ambos campos. 
Keywords:

Women's/Gender/Studies; history; queer theory; feminist theory; time; archive.

\section{Abstract}

In the 1980 s Women's/Gender/Studies and Women's/Gender/History seemed closer to each other than they subsequently have become. Here I ask, Can Women's/Gender/ Feminist Studies be historicized and can history be theorized by queer and feminist theory in ways that illuminates the past on its own terms and not merely speak to the present or the future? I consider citational practices and subfields in light of larger intellectual trends, especially work in Black feminism. Exploring this question, I turn to how each field thinks about time and the archive, terms that function as analytics to illuminate questions of power and authority, identity and agency, but deployed by interdisciplinary feminist scholars in ways not always legible to traditional historians. I end with both reconciliation and alternative endings in light of attacks on both fields.

Como historiadora de las mujeres y de género en el Departamento de Estudios feministas, antes llamado programa de Estudios de las mujeres, he tomado consciencia, en el espacio de la clase, en tanto que académica y dentro del ámbito profesional, de las paralelas y divergentes trayectorias de la historia de las mujeres/historia de género y de los Estudios de las mujeres/Estudios de género como sitios de producción de conocimiento sobre mujeres, género, sexualidades y sus intersecciones con raza, nación y espacio. En los años ochenta estos campos parecían más cercanos que en las décadas subsiguientes, a pesar de la presencia de muchxs historiadorxs -entre lxs que me incluyo en departamentos de mujeres, género, sexualidad y estudios feministas. Si bien fue central para la formación interdisciplinaria de los estudios de las mujeres/estudios de género/estudios feministas, la historia hoy resulta marginal a sus intereses. De todos modos, estos campos han incorporado categorías propias de la historia como disciplina: el tiempo y el archivo. Aquí me propongo explorar esos términos en relación a los estudios feministas.

Me pregunto, ¿pueden el archivo y el material, el pasado como cosa y como cuerpo, reconfigurar el presente? ¿Pueden historizarse los estudios de las mujeres/estudios de género/estudios feministas y puede la historia ser teorizada por la teoría queer y la teoría feminista en modos que iluminen el pasado en sus propios términos, tal como todavía suelen hacer lxs historiadorxs, y no simplemente dirigirse al presente o al futuro? Dejando de lado metáforas del matrimonio y el parentesco, ¿deberíamos salvar esta relación? ¿Cómo podríamos hacerlo? ¿O, de acuerdo con la idea que sugiere Joan Scott y de la que se apropia la historiadora australiana Joy Damousi, la historia es una disciplina que ha absorbido la investigación sobre mujeres y género como categoría de análisis y, así, ha separado el estudio de los movimientos sociales que brillaron en los años sesenta y setenta y que permanecen presentes en los estudios feministas, aunque en formas diferentes respecto de los movimientos originales?

En su lección inaugural para la conferencia anual de la National Woman Suffrage Association (NWSA) de 2001, la teórica feminista Robyn Wiegman interrogó críticamente cuál es el tiempo de los Estudios de las mujeres e insinuó, muy a su pesar, que ese tiempo es el ahora, o bien un largo presente que se extiende hacia atrás una o dos décadas, pero que se trata de un campo fundamentalmente presentista. Lxs estudiantes desean pensarse a sí mismxs, sus circunstancias, y cuando quieren estudiar los orígenes, se refieren a sus propios orígenes. He percibido que, salvo escasas excepciones, ese es el caso de lxs estudiantxs que se acercan a clases de estudios feministas en University of California Santa Barbara (UCSB). Pero este deseo no es negativo. Tal como me lo han recordado recientemente, para quienes pertenecen a grupos que en el pasado han sido suprimidos o negados, tal exploración no es solo vital sino también productiva. 
La historia de las mujeres ha sido central para la fundación de los estudios de las mujeres. Hemos desarrollado nuevos cursos organizados cronológicamente (desde 1890 hasta el presente), de acuerdo con contenidos (mujeres y trabajo) y espacialidades (mujeres en el sur, historia de las mujeres británicas) en la historia de las mujeres. Además, con frecuencia, historiadorxs de las mujeres han enseñado las clases introductorias de estudios de las mujeres, donde se comparan las vidas y status de varios grupos de mujeres en diversos tiempos y espacios, diferentes al momento contemporáneo. Hemos identificado que el pasado es un país diferente y lo hemos explorado, pero cuando entramos a una clase de estudio de las mujeres, ejercemos la historia con un propósito. Como lxs estudiantxs, la historia mira al pasado para avanzar, observa el tiempo presente y el tiempo pasado que se manifestarán en el tiempo futuro. La historia de los movimientos de mujeres ha sido particularmente importante en la búsqueda de aquellos pasados utilizables que permearon los inicios de los estudios de las mujeres y que motivaron su giro hacia la historia. Procuramos explicar cómo llegamos a lo que parece ser un presente integrado. Entonces fue importante aprender acerca de las conexiones y fisuras entre el abolicionismo de la esclavitud y los derechos de las mujeres mientras contemplábamos el vínculo entre derechos civiles, black power y el nuevo feminismo de los sesenta y setenta, así como también enfrentamos los desafíos de las mujeres negras hacia formas del feminismo hegemónico. Recuperamos la agencia de niñas trabajadoras, empleadas domésticas esclavizadas, jóvenes costureras huelguistas, inmigrantes transfronterizas, travestis. Documentamos la discriminación construida dentro de la ley y la política social. Nos comprometimos con los estudios de las contribuciones que las mujeres han hecho en la historia (había mujeres allí también), reconceptualizamos tanto la periodización cuanto el carácter de los grandes acontecimientos, postulamos una historia intrínseca a las vidas de las mujeres, basadas en el curso de la vida y otorgamos una historia a las prácticas sexuales y a las identidades. También hicimos lecturas de género de la política, la economía y las fuerzas sociales. Gerda Lerner y Joan Scott, en modos que contrastan entre sí, produjeron avances historiográficos (jaunque Scott probablemente lo negaría!) que establecieron progresos de la simplicidad hacia la complejidad, de la contribución hacia las condiciones propias, de las mujeres al género. Al margen, Lerner en su producción de fines del siglo XX, consideró que el giro cultural perturbaba, en tanto nos distanciaba de lo económico y de la noción de clase, pero el contexto histórico en el que escribimos da forma a interrogantes que solemos hacernos y que ciertamente determinaron sus movimientos personales e intelectuales, así como los nuestros.

Para quienes no lo sepan, Lerner fue refugiada durante el Nazismo, es una judía que huyó de Viena, comunista durante algún tiempo, radical siempre; ama de casa izquierdista, activista y escritora por los derechos civiles, relacionada al Partido Comunista. Condenó el enfoque de Betty Friedan en la "mística femenina" por "concentrarse únicamente en los problemas de las mujeres universitarias de clase media", en lugar de orientarse a las "mujeres trabajadoras, especialmente a las mujeres negras", quienes "trabajan en condiciones de desventaja impuestas por la mística femenina, y bajo las aún más acuciantes desventajas de la discriminación económica". Su texto The Lady and the Mill Girl (1973), su trabajo con el feminismo abolicionista de la esclavitud, y su libro Black Women in White America (1972) se gestaron en su posicionamiento político, pero terminó sustituyendo género por clase como el principal factor determinante de las vidas de las mujeres en The creation of patriarchy (1986).

Hoy la globalización y la feminización del trabajo han asumido una nueva relevancia; algunxs entre nosotrxs llevamos a cabo análisis culturales de clase. La violencia racializada del estado y los modos en que trata a inmigrantes sin duda impactan ambos campos, del mismo modo en que los han impactado otros asuntos políticos recientes, como la trata, las identidades de género y el matrimonio entre personas del mismo 
1. Amicus curiae (amigo de la corte o amigo del tribunal) es una expresión latina utilizada para referirse a presentaciones realizadas por terceros ajenos a un litigio, que ofrecen ajenos a un litigio, que ofrecen ( a algún punto de derecho $u$ otro aspecto relacionado, para colaborar con el tribunal en la resolución de la materia objeto del proceso.

2 Original en inglés: Home care workers. sexo, los cuales generan investigación histórica y descansan sobre la autoridad de la historia en la esfera pública. De hecho, en ocasiones, historiadorxs de las mujeres y el género han jugado roles sustanciales en varios amicus curiae ${ }^{1}$ ante la Corte Suprema en los Estados Unidos, entre los que se incluyen los casos de Nancy Cott y George Chauncey sobre matrimonio entre personas del mismo sexo; Linda Kerber y un grupo de historiadorxs de la ley y el trabajo, entre quienes me encuentro, intervinieron en un caso sobre aborto y regulación clínica que visibilizó sentidos de protección para las libertades de las mujeres (muchxs de esxs historiadorxs trabajaban en un caso de licencia por maternidad y paternidad hace una década) y mi coautora Jennifer Klein y yo, en un caso que debatió el rol de trabajadorxs domésticos que ejercen tareas de cuidado ${ }^{2}$ como empleadxs públicxs (un caso sobre el "derecho al trabajo" que intentó usar a madres que ejercían tareas específicas de cuidado en sus hogares para destruir la sindicalización del sector público).

Antes que lamentar una carencia, hay quienes desestiman esta temprana influencia de la historia que, con su promesa de progreso, ubica los Estudios de las mujeres en una trayectoria intelectual, lo cual produce consecuencias hegemónicas. Al escribir en 2011 para Feminist Studies, Amy L. Brandzel insistió acerca de los riesgos que conlleva privilegiar la historia, sobre todo la historia del feminismo, porque tal gesto resulta en una reificación de la normativa blanca de acuerdo con la que el ciudadano sujeto es la fuerza del progreso en la historia. Incluso en la medida en que textos y voces, acciones y experiencias de diversas índoles producidas o vividas por otrxs se mezclan y se remueven, la narrativa, sostiene Brandzel, se mantiene constante: la expansión de la inclusión y la entrada a la ciudadanía, sin un reconocimiento pleno de las dinámicas del poder y sin una reconfiguración de la historia que contamos, no cambian su lógica. Por lo tanto, estamos obligadxs a progresar, renunciar o volvernos retórica, tal como ha subrayado la teórica británica Clare Hemmings. Brandzel nos desafía a quienes enseñamos e investigamos la historia de las mujeres a "no pensar en la historia y la futuridad del feminismo como algo que espera a ser descubierto, a volverse inteligible, ni, de ninguna manera, algo rectificable, sino como un proyecto necesariamente inmerso en (y por lo tanto reflector de las relaciones entre) subjetividad, relaciones de poder y producción de conocimiento". Más allá de la narración, herramienta de lxs historiadorxs, deberíamos comprometernos con la deconstrucción. Muchxs historiadorxs percibirán un espantapájaros: concuerdan en que expandir los relatos a través de la inclusión no es adecuado, pero al mismo tiempo, caracterizaciones como la de Brandzel, arriesgan una petrificación del dinamismo de la historia como disciplina que no dejará de tomar prestados elementos de otras áreas en su búsqueda de mejores explicaciones o de ninguna explicación en absoluto. De hecho, la aprobación del derecho a sufragio para las mujeres estuvo en primera instancia fuertemente ligada al imperialismo y a las leyes de Jim Crow y, luego, a la masacre que fue la Segunda Guerra Mundial; sabemos que la expansión de la ciudadanía no ha sido un proceso impoluto. En mi opinión, demasiadxs docentes e investigadorxs de los estudios de mujeres/estudios de género/estudios feministas no leen lo suficiente acerca de las nuevas historias.

Existen claras diferencias epistemológicas y metodológicas entre la historia de las mujeres y los Estudios feministas. La divergencia puede haberse desarrollado a partir de la expansión de sendos proyectos hacia la formación de campos en sus propios términos; al codificarse y crecer exponencialmente las publicaciones al respecto, generaron nuevas cadenas de la historiografía y la canonización. En los Estados Unidos, pocxs entre quienes ofrecen estudios o incluso cursos de grado en historia de las mujeres enseñan Robyn Wiegman, Clare Hemmings, Kimberly Crenshaw, Sara Ahmed, Jasbir Puar, Chandra Mohanty o Gloria Anzaldua, aunque formen parte del "canon" de los Estudios feministas. Son teóricas de otras disciplinas, no historiadoras. Únicamente Joan Scott aparece con cierta regularidad entre estxs supuestxs 
"historiadorxs" y no ocurre en cursos sobre historia de los Estados Unidos. En general las clases de estudios feministas no enseñan historia. Se enseñan, como mucho, mis escritos menos históricos y lo hacen solamente sociólogxs que analizan las tareas de cuidado y procuran un pasado que les sea útil al dictar sus clases o académicos que investigan las relaciones de trabajo.

Por su puesto, ninguno de estos campos es estático. Tanto la historia de las mujeres cuanto los estudios de las mujeres se sometieron a influencias intelectuales más generales, tales como el género en tanto que categoría de análisis, los estudios interdisciplinarios y críticos sobre raza, el postmodernismo y el giro lingüístico, los estudios culturales, el poscolonialismo, el transnacionalismo y la globalización. Ambos campos se han movido desde las mujeres como entidad fáctica hacia el género como significante de relaciones de poder, y ninguno de ellos ha abandonado totalmente a las mujeres como esencia corporizada y simbólica, aunque la transición de la historia social hacia los estudios culturales haya significado que a veces no se trabaje con la categoría 'mujeres' sino con feminidades y masculinidades, lo femenino y lo masculino, y el género como productor de pliegues en lo híbrido. En muchas áreas de la historia, el movimiento es, tal como afirmó Nancy Green en un artículo de 2012 sobre historia de las migraciones, de los hombres a las mujeres al género; de experiencias de la historia social al impacto de los regímenes de género antes, durante y después de migrar/establecerse, proceso en el cual existen políticas de estado que se fusionan con presupuestos del orden de género acerca de la familia, el trabajo y la sexualidad. Pero a pesar de los movimientos al interior de los estudios históricos, las resistencias mantienen el modo tradicional de entender cómo se narran y se entienden los documentos: el grupo de estudios sobre enseñanza de la historia de mujeres de los Estados Unidos que se reúne anualmente en University of California Los Angeles (UCLA), por ejemplo, consideró que el estudio no narrativo Somebody's Children: The Politics of Transnational and Transracial Adoption (2012) en el que Laura Briggs analiza la adopción y los daños que produce para niñxs afroamericanxs, indígenas y latinoamericanxs en Estados Unidos no es histórico; Laura es una historiadora posicionada como académica de los estudios de las mujeres/estudios de género/ estudios de la sexualidad. Existen maravillosos usos de la Historia que usa historia para otros géneros. The Traffic in Women, de Laura Kang, es un buen ejemplo de ello ya que cuestiona interdisciplinariamente, desde los estudios de asiáticos en Estados Unidos, y rastrea usos de mujeres "de consuelo" (comfort women) y formas tempranas de la trata de mujeres asiáticas como parte del Imperio Británico en la década de 1920, pero no está escrito desde los parámetros establecidos para lxs historiadorxs. En la misma línea se ubican el análisis de clase que propone Miranda Joseph de la financierización y la generización en Debt to Society: Accounting for Life Under Capitalism (2014) y The Afterlife of Slavery: Human Reproduction in Biocapitalism, el reciente trabajo de Alys Eve Weinbaum que abordaré brevemente aquí. De hecho, hay signos de que sendas esferas se mueven en direcciones que prometen futuras convergencias a través de intereses comunes en la globalización, el transnacionalismo y la política económica, entre abordajes multi metodológicos y multi teóricos para organizar la interpretación y la investigación. Lxs historiadorxs historizan sensaciones, emociones y otros afectos dentro del marco de un giro afectivo más general.

Aun así, al leer "historia" y al leer "estudios feministas", suelo sentir una disyunción fundamental respecto de la presentación y las condiciones del análisis. Pero eso no significa que los componentes cruciales de la historia el cambio y la continuidad a través del tiempo y dentro de espacios específicos estén ausentes en los estudios feministas. Lxs académicxs de los estudios feministas con frecuencia leen la historia con la intención de construir teoría y no con el afán de sumar debates interpretativos dentro de los estudios históricos. Me simpatiza mucho ese proyecto, aunque reconozco que es distinto a la producción en historia. ¿Pueden la narrativa y la deconstrucción 
3 Sistema de trabajo en condiciones de esclavitud llevado a la práctica en estados sureños de Estados Unidos explícitamente autorizado por la Enmienda XIII (1865). Se trata de encarcelamiento de convictos para esclavizarlos como mano de obra no remunerada y de manera involuntaria en diferentes plantaciones $y$ taria en diferentes plantaciones y
corporaciones. Los esclavizadores privados, y no el estado, eran responsables de la alimentación, la vestimenta y la vivienda de estos convictos esclavizados. coexistir en ambos campos? La historia permanece más atada a la narrativa que los estudios feministas, pero no todxs lxs historiadorxs presentan la narrativa de la misma manera; pensemos en la microhistoria, la historia personal y la macrohistoria.

En este punto destaco el último libro publicado de Alys Eve Weinbaum, The Afterlife of Slavery: Human Reproduction in Biocapitalism. Desde la crítica literaria y los estudios de género, esta académica procura revelar la persistencia de la esclavitud, especialmente en términos de reproducción y crianza, en la venta de seres humanos, partes de cuerpos humanos o gestación subrogada en la actualidad. Se interesa por la genealogía, pero también por la persistencia de una "episteme de esclavitud" que ha tolerado expropiaciones reproductivas, a lo que denomina "nexo de esclavitud/subrogación". Lee el pasado con un ojo hacia el futuro, como anticipo del presente y como residuo en el presente. No obstante, como buena historiadora, sitúa textos del feminismo negro de los años setenta, ochenta y noventa en sus contextos de producción, a partir de su "distintiva filosofía de la historia", para discutir cómo esos trabajos enfatizaban el rol central de las mujeres negras en la resistencia de los trabajadores negros contra la esclavitud, no solamente ante el capitalismo racializado en su momento neoliberal, sino también ante el biocapitalismo que se ha desarrollado durante las últimas décadas. Weinbaum vuelve a textos ficcionales como Beloved (1987) de Toni Morrison "para figurarse el rechazo de mujeres esclavas a la expropiación sexual y reproductivo en y para el presente y el futuro" (Weinbaum, 2019: 64). En efecto, sostiene que Morrison tuvo en mente el caso de Joanne Little, una mujer negra encarcelada que en 1974 mató a un guarda que la amedrentaba sexualmente para escribir Beloved. Weinbaum afirma que "las feministas negras complementaron un archivo restringido, enmendaron la historiografía dominante y testimoniaron la persistencia del trauma histórico de la esclavización para mujeres" (Weinbaum, 2019: 64). Esto es, "imaginaron una historia de rechazo y desacuerdo y propusieron una inversión porque vieron la insurgencia violenta de mujeres esclavizadas como algo relevante para las luchas contemporáneas dirigidas a redefinir la situación de las mujeres" (Weinbaum, 2019: 64) hoy.

Lxs historiadorxs han apelado a este tipo de relatos para comprender la verdad cuando los hechos se presentan escasos y dispersos. Así Sarah Haley en No Mercy Here:Gender, Punishment, and the Making of Jim Crow Modernity (2016), en sintonía con Weinbaum, recorrió la producción de la crítica literaria Saidiya Hartman para reconstruir las subjetividades de mujeres encarceladas mediante una lectura oblicua de casos judiciales, investigaciones gubernamentales e informes institucionales, petitorios, relatos periodísticos y correspondencias. Captura así el sentimiento, el olor, el dolor y la insensibilidad del encarcelamiento, pero también la amistad forjada en medio de esas condiciones brutales, como modo de revelar resistencias. Es decir, genera un marco alternativo para el convict lease system ${ }^{3}$ por rechazar la abyección y abrazar la femineidad negra más allá de la respetabilidad. Nombra allí la violencia sexual en el corazón del estado racializado. Este libro es un buen ejemplo de la riqueza de los archivos, pero también es una muestra de cómo lxs historiadorxs debemos prestar atención a persistencias del pasado en el presente, tales como las actualizaciones carcelarias de las leyes de Jim Crow.

A partir de aquí, consideraré el tiempo y el archivo de acuerdo con las perspectivas de los estudios de mujeres/estudios de género/estudios feministas. Estos términos funcionan como categorías de análisis que iluminan interrogantes sobre poder y autoridad, identidad y agencia; pero el modo en que académicxs feministas interdisciplinarixs los despliegan no siempre es legible desde los mismos códigos en que los aplican la mayoría de lxs historiadorxs. Y, sin embargo, quienes estamos en ambos campos nos movemos entre esos modos de pensamiento. 
Lxs historiadorxs segmentamos el tiempo en siglos, décadas, eras; la longue dureé, pero también, en fechas específicas. Los estudios feministas han tomado esa preocupación por el tiempo, con frecuencia direccionada hacia futuros queer o hacia el fin de la historia, ya sea como utopía o distopía. ¿Qué es el tiempo queer? Elizabeth Freeman en el vol. 23, $\mathrm{n}^{\circ}$ 2-3 de $G L Q$ (2017) nos sugiere:

[R]efigurémonos lo queer como conjunto de posibilidades producido fuera de la diferencia temporal e histórica, o veamos la manipulación del tiempo como modo de producción de cuerpos y relacionamientos... En efecto los estudios queer se cruzan con la teoría crítica sobre raza y los estudios poscoloniales porque entienden que aquello que aún no figura en los registros históricos y aquello que todavía no es culturalmente legible, se encuentra con frecuencia en formas corporizadas, no racionales[...]. (Freeman, 2017: 159)

Paralelo a su concepto de "aquello que aún no ha ingresado" que los estudios queer iluminan, se presentan la discusión de Dipesh Chakrabarty sobre (a)temporalidad subalterna y cambio climático y el clásico Work, time and Industrial Discipline de E.P. Thompson, el cual plantea formas alternativas de entender la temporalidad agraria desde el tiempo industrial. Por su parte, Bettina Aptheker ha escrito sobre el tiempo de las mujeres, que podríamos traducir por tiempo doméstico, para generar un sentido menos esencialista de los ritmos en los que más que el género, se reflejan conexiones de labor y rutinas, aunque el tiempo del trabajo doméstico se dimensione de acuerdo con clase, raza y género en múltiples aspectos. Estas son nociones alternativas de tiempo paralelo, que todavía no forma parte del registro histórico, pero que debemos insertar en ese registro mediante el énfasis en su significancia, las emociones y sentimientos, prácticas relegadas al terreno de lo no nombrado.

Para lxs historiadorxs, el futuro es menos preocupante que aquello que emerge desde el pasado. Durante mucho tiempo parafraseé a Marx en el programa de las materias a mi cargo: las personas hacen la historia, pero no la crean en circunstancias que puedan elegir, sino en aquellas que han heredado del pasado. El pasado debe abordarse en sus propias condiciones (¿a quién pertenecen esas condiciones?) incluso si nuestros cuestionamientos nacen del presente. Entonces, aunque el pasado puede alumbrar el presente y contribuir en dirigirnos hacia el futuro, no podemos leer el pasado como si fuera el ahora, gesto que distorsionaría y oscurecería aquello que puede ser realmente importante y lo perderíamos por no ser legible desde las lentes contemporáneas. Pero la propia distancia del pasado nos ofrece caminos no explorados, visiones alternativas y lecciones aprendidas. Un sentido de la diferencia se manifiesta en el siguiente pasaje de The Archival Turn in Feminism: Outrage in Order (2013) de la especialista en Estudios culturales, Kate Eichhorn:

El depósito de chatarra... no es sitio para rechazar ni para el rechazo, sino un complejo espacio donde el pasado se acumula en el presente como recurso que debe incorporarse y repudiarse, minarse y reciclarse, descartarse y redistribuirse. Entonces, el depósito de chatarra del feminismo es un sitio de lo abyecto $\nabla q u e$ como tal debe expulsarse, pero sin el cual no podemos vivir囚 y en simultáneo, un patio de juegos, un refugio, un escenario de innovación, humor, esperanza y anhelos. (Eichhorn, 2013: 29)

La autora demanda "tocar la historia y comprometerse con su construcción" como algo central para ser académicas feministas (Eichhorn, 2013: 54). Esta noción es similar a la de Freeman, quien nos exhorta a comprometernos con la "temporalidad drag" e impregna lo histórico de erotismo, como si la memoria fuera evocación que decanta en sensaciones. Tal análisis nos coloca de nuevo en el tiempo actual de los estudios de mujeres. 
Consideremos, para finalizar, el archivo. Los estudios feministas lo han descubierto recientemente. Muchxs académicxs mencionan la creación o el montaje de un archivo. Pero la elección de un archivo para desplegar o trabajar una teoría no es lo mismo que la producción de sentido a partir de las huellas del pasado. Por su puesto, los propios archivos son construcciones que dependen de coleccionistas, reservas, autorxs. Son verdades y ficciones parciales. Aun así, lxs historiadorxs al visitarlos se contagian una "fiebre de archivo" (Carolyn Steedman habla de una fiebre real, producida por el polvo y los viejos materiales con que se encuadernaban libros), una excitación por encontrar, por los trazos de aquello que alguna vez fue: el alfiletero aprovechado por Elizabeth Keckley para coser el vestido de Mary Todd Lincoln; los libros de actas en los que Reagan se descargaba contra César Chávez, Angela Davis y otrxs; un mechón de cabello; el expediente de un caso de trabajo social sobre el cual se genera la idea de un libro provienen del archivo. Sensación histórica. El contacto con el pasado, esa voz que se anhelaba escuchar, el hallazgo un documento, eso es lo que encontramos en el archivo o lo que construimos entre esa parva de restos, para usar palabras de Eichhorn. Podemos diferenciar entre el romanticismo, que creía en la recreación del pasado (fantasía del origen) y el positivismo, que se imbricaba con una cientificación (fantasía del poder), movimientos que autenticaban el pasado. En nuestra edad de la deconstrucción, vemos con mayor nitidez. De todas maneras, no puedo evitar invitar a mis estudiantes a probar el archivo, a deleitarse en las colecciones especiales, a sentir la conmoción y el misterio de la incompletud. ¡Y, en simultáneo, Pat Cohen dirige a sus estudiantes hacia $<\mathrm{http}$ :/Www.ancestry.com $>$ y otras bases de datos!

Pero ¿es el archivo una reliquia del pasado o del presente? En este punto, los ahora estudios feministas, redimensionan el archivo así como el tiempo. "El archivo aparece, no como lugar para recubrir el pasado sino como forma de religarse con algunos legados epistémicos y traumas que prensan el presente" anota Eichhorn (2013: 5). Al historizar esta vasta apropiación y redefinición de los archivos, apunta hacia el impacto del neoliberalismo el socavamiento de la agencia en el presente mediante sus procesos de mercantilización, financierización, privatización y las inequidades y precarizaciones resultantes que hace del pasado un lugar en el cual excavar alternativas; no meramente un espacio para la nostalgia sino también una manera de legitimar reclamos para el presente y el futuro, para comprometerse con lo que la filósofa Wendy Brown, en un eco foucaultiano, llama "política genealógica".

Sin embargo, los archivos oficiales no suelen organizarse según nuestras propias búsquedas. El método histórico tal como se ha desarrollado en la modernidad occidental depende de la manutención de registros en reservas oficiales que albergan acontecimientos y hechos de la nación, así como también la verificación y el análisis de la documentación. Los archivos funcionan como parte de la clasificación y el registro disciplinarios de datos, tareas vertebrales a la constitución del estado moderno y colonial, a la creación de la ciudadanía y los sujetos, y al poder, como parte de las dinámicas de conocimiento poder de la modernidad y su definición del tiempo. Por lo tanto, algunos documentos encajan y otros se rechazan dependiendo del esquema clasificatorio, la trayectoria de significaciones implicada. Los archivos incluyen la ley, regulaciones, secretos y nomenclaturas (un "orden de las cosas" ¿quién lo dispone? ¿qué cosas? difícilmente natural). De acuerdo con Eichhorn y muchxs otrxs, el archivo se convierte en sitio de "estratégica restructuración que crea actores presentes que nunca antes habían tenido la oportunidad de intervenir en un determinado campo, pero que, en muchos niveles, de todas maneras, ocupaban cómodamente ese mismo campo" (Eichhorn, 2013: 90), una posible práctica de la genealogía.

¿Quién tiene poder para decidir qué vale y qué historia se cuenta? Lxs historiadorxs de la subalternidad han desplazado poderosamente nuestro concepto de archivo al recurrir a la ficción para reimaginar los archivos... e incluso los usos del pasado. A 
este respecto, destaco el trabajo de Haley, pero también Ties that Bind: The Story of an Afro Cherokee Family in Slavery and Freedom (2005), donde Tiya Miles hace uso de Beloved de Tom Morrison tanto cuanto de su propia imaginación para concebir desde la emoción y el sentir allí donde el registro preservado era silencioso. En el centro de su historia está Doll, una africana esclavizada, compañera de vida de Shoe Boots, guerrero cheroqui que la ha comprado. ¿Y qué piensa ella? La historia está organizada, de manera similar a Beloved, temáticamente (esclavitud, matrimonio, maternidad), como meditación entre presentes y pasados cercanos para alcanzar la violencia y las ataduras que ligan este tipo de constelaciones familiares.

Para concluir: no toda historia de las mujeres/historia de género es feminista, pero la mayor parte de los estudios de mujeres/estudios de género/estudios feministas lo son. 'Mujer' como categoría no opera del mismo modo que género y feminismo. Al significarnos, nosotrxs mismxs como estudiosxs feministas, procuramos atravesar la cuestión acerca de qué cuerpos importan (y se materializan), aunque la historia trabaje casi siempre con cuerpos reales, imaginados y simbólicos.

Ahora bien, nos encontramos frente a ciertas preguntas: ¿Qué feminismos? ¿Practicados por qué feministas? ¿Cómo continuamos produciendo nuestros campos de estudio? ¿Qué tomamos de cada disciplina, si ya no trabajan fusionadas? Admitimos que la historia de las mujeres y la historia de género, cada cual, se arraigan dentro de un gran marco que, siempre caprichoso, redirige nuestras raíces y las alimenta con cualquier herramienta que ayude a interpretar, aprovecha la teoría feminista y la teoría queer y se encuentra con otras disciplinas. Por su parte, los estudios feministas maniobran dentro y entre los deseos, el poder y las condiciones que dan forma a la universidad y al mundo, al hablar y proponer alternativas o simplemente otras sendas en oposición a una soberanía exteriorizada y políticas represivas en el hogar, por lo que estimulan el espíritu de las rebeliones aquí, ahora, las que están en la memoria y aquellas a ser realizadas. En nuestras historias surgen perspectivas; en nuestras perspectivas, la justicia social es posible.

Pero propongo aquí un final alternativo: tal vez tanto la historia de las mujeres/historia de género cuanto los estudios de mujeres/estudios de género/estudios feministas tengan un futuro inhóspito en términos de permanencia en la corporación universitaria que empuja a lxs estudiantes hacia las ciencias exactas y naturales, la tecnología, la ingeniería y las matemáticas. Cuando carecemos de financiamiento por no ser capaces de obtener subvenciones públicas, financiamientos privados ni alumnxs que dispongan de altos recursos, ¿habrá tesis doctorales que produzcan nuevos conocimientos para la historia feminista y el conocimiento de género? Sentimos la falta de reemplazo de una generación de pionerxs que se han retirado o han fallecido. Sin embargo, no quiero detenerme en ese punto. Me inspira la propuesta de la historiadora británica Kathryn Gleadle en su artículo publicado en 2013 por Women's History Review, donde propone una red de mujeres historiadoras o, mejor dicho, una serie de redes. Debemos considerar la IFRWH como uno de esos árboles con múltiples raíces no lineales. Algunas de estas redes se extienden hacia los estudios feministas, otras no, pero conectan a lxs académicxs con disciplinas como la sociología y la literatura. Por lo tanto, en última instancia, tengo las esperanzas de que la interacción y las conversaciones a través de los bordes del tiempo, el espacio y el campo nos guíen hacia una nueva academia, más productiva y con mayor impacto. 


\section{Q Bibliografía}

"Aptheker, Bettina. Tapestries of Life. Women's Work, Women's Consciousness, and the Meaning of Daily Experience. The University of Massachussetts Press, 1989.

» Brown, Wendy. Politics out of History. Princeton University Press, 2001.

»Boris, Eileen y Klein, Jennifer. Caring for America. Home health workers in the shadow of the Welfare State. Oxford University Press, 2012.

»Briggs, Laura. Somebody's Children: The Politics of Transnational and Transracial Adoption. Duke University Press, 2012.

»Brandzel, Amy L. "Haunted by Citizenship: Whitenormative CitizenSubjects and the Uses of History in Women's Studies," Feminist Studies 37 (Otoño 2011): 503-33.

»Cott, Nancy. Public Vows. A history of Marriage and the Nation. Harvard University Press, 2002.

»Chauncey, George. Why Marriage: The History Shaping Today's Debate Over Gay Equality. Basic Books, 2005.

"Eichhorn, Kate. The Archival Turn in Feminism: Outrage in Order. Temple University Press, 2013.

»Freeman, Elizabeth. "Introduction” to 'Queer Temporalities' Issue. GLQ A Journal of Lesbian and Gay Studies 13, n’2/3 (2007): 159-175.

»Friedan, Betty. The Feminine Mystique. W. W. Norton, 2001

» Gleadle, Kathryn. "The Imagined Communities of Women's History: current debates and emerging themes, a rhizomatic approach", Women's History Review, vol 22, ${ }^{\circ} 4$ (Agosto 2013), pp.524-540.

"Green, Nancy. "Expatriation, expatriates and Expats: the American Transformation of a Concept", American Historical Review, 114 (2), 307-328.

»Hemmings, Clare. Why stories matter. The political grammar of feminist theory. Duke University Press, 2011.

" Haley, Sara. No Mercy Here: Gender, Punishment, and the Making of Jim Crow Modernity. The University of North Carolina Press, 2016.

" Joseph, Miranda. Debt to Society: Accounting for Life Under Capitalism. University of Minnesota, 2014.

» Kang, Laura. The Traffic in Women, Duke University Press, Forthcoming 2020.

»Lerner, Gerda. The Lady and the Mill Girl, Warner Modular Publications, 1973.

"Lerner, Gerda. Black Women in White America: A Documentary History, Vintage Books, 1992.

»Lerner, Gerda. The creation of patriarchy, Oxford University Press, 1986.

»Miles, Tiya. Ties that Bind: The Story of an Afro®Cherokee Family in Slavery and Freedom. University of California Press, 2005.

» Morrison, Toni. Beloved. Vintage Classic, 2010.

"Palmer Thompson, Edward. "Time, Work Discipline and Industrial Capitalism", Past and Present, 38, no. 1279 (1967), 56-97. 
»Steedman, Carolyn. Dust: The Archive and Cultural History. Rutgers University Press, 2002.

"Weinbaum, Alys Eve. The Afterlife of Reproductive Slavery: Biocapitalism and Black Feminism's Philosophy of History. Duke University Press, 2019. 
